\title{
Incongruence of neurophysiological manifestations as reflection of disturbances in synaptic pruning of the cortex in paranoid schizophrenia
}

Andrei Yurevich Arkhipov ( $\square$ arhip76@bk.ru )

RAS Institute of Higher Nervous Activity https://orcid.org/0000-0001-8301-7921

Malik Kubanichbekovich Nurbekov

FSBI Institute of General Pathology and Physiopathology of Russian Academy of Medical Sciences:

FGBNU Naucno-issledovatel'skij institut obsej patologii i patofiziologii

Valeria Borisovna Strelets

RAS Institute of Higher Nervous Activity

\section{Research Article}

Keywords: Paranoid schizophrenia, Evoked potentials, Paradox effect, Synaptic elimination, RELN gene

Posted Date: September 23rd, 2021

DOI: https://doi.org/10.21203/rs.3.rs-928057/v1

License: (a) (1) This work is licensed under a Creative Commons Attribution 4.0 International License.

Read Full License 


\title{
Incongruence of neurophysiological manifestations as reflection of disturbances in synaptic pruning of the cortex in paranoid schizophrenia
}

\author{
Arkhipov Andrei Yurevich, ${ }^{1, \dagger}$ Nurbekov Malik Kubanichbekovich ${ }^{2}$ and Strelets Valeria Borisovna ${ }^{1, \uparrow}$ \\ ${ }^{1}$ Laboratory of human higher nervous activity, Institute of Higher Nervous Activity and Neurophysiology \\ Russian Academy of Sciences, Moscow, Russia \\ 2 Laboratory of regulation of reparative processes, FSBI Institute of General Pathology and \\ Physiopathology of Russian Academy of Medical Sciences, Moscow, Russia
}

Correspondence to: Arkhipov Andrei Yurevich, MD

Institute of Higher Nervous Activity and Neurophysiology Russian Academy of Sciences, Laboratory of Human Higher Nervous Activity, 5A Butlerova str., Moscow, 117485, Russia

E-mail: arhip76@bk.ru 


\begin{abstract}
Current study provides a comprehensive analysis of neurophysiological and molecular genetic data concerning the etiology of hallucinatory-paranoid syndrome in schizophrenia. The level of cortical excitation and inhibition was determined by analysis of event-related potential (ERP) parameters: latency and amplitude of intermediate (P200) and late (P300, N400) ERP components. The results showed the impossibility to determine the level of different cortical areas activation during perceiving stimuli of different significance in patients with hallucinatory-paranoid syndrome due to unidirectional shift of both ERP parameters to significant stimuli. The observed simultaneous increase of both amplitude and latency of these components in frontal regions is associated with an excessive number of aberrant synapses, and a decrease in the central regions is associated with synaptic deficit, this is due to disturbances in synaptic pruning of the cortex. Thus, the inhomogeneity of number and quality of synapses in central and frontal cortical areas was revealed, caused by inhomogeneity of their elimination process, which may be an essential condition for hallucinatory-paranoid syndrome emergence. An epigenetic analysis was also performed to assess the methylation level of gene expressing an extracellular matrix protein and playing a key role in neural connections distribution in ontogenesis. The detected epigenetic disorders in form of demethylation of RELN gene cause pathological synaptic pruning and elimination diversity in different brain areas.
\end{abstract}

Keywords: Paranoid schizophrenia; Evoked potentials; Paradox effect; Synaptic elimination; RELN gene

\title{
Introduction
}

An alteration of synaptic elimination may form a basic pathology for neurophysiological abnormalities in schizophrenia and correlate with the clinical picture of the disease (Feinberg 1990). Comparing the results of the study of postnatal 'synaptic pruning' mechanisms (Feinberg 1983) with the clinical picture of the disease, Feinberg (Feinberg 1983; Keshavan 1994) formulated a radically new theory of schizophrenia etiology, suggesting that the disease can occur as a result of pathological synaptic pruning. However, Feinberg's studies (1983) did not describe the exact pathophysiological mechanisms of the elimination process disturbance, but only suggested the possibility of synaptic connections number increase or decrease and pathological synapses formation.

Feinberg hypothesis (1983) was extended by Hoffman and Dobscha (1989), and as well by Jernigan and colleagues (1991), who suggested that excessive pruning of collateral axons in the prefrontal cortex (PFC) leads to clinical manifestations of schizophrenia.

Most studies relating disorders of synaptic elimination in schizophrenia correlate them with structural and morphological indicators: changes in cortical thickness (Wannan 2019); decrease in size of neurons in limbic, temporal and frontal regions (Higley 2003; Borgwardt 2008), abnormal density of 
dendritic spines in the cortex, altered cytoarchitecture (Glausier 2013) (which may be associated with abnormal migration, differentiation of neurons or a reduction of cell number).

Molecular-genetic studies attempt to find a correlate of these structural brain alterations in structural gene violations that provoke impairments in expression associated with synaptic function, energy metabolism, immune system activation, and oligodendrocyte transcripts (Mauney 2015; Fromer 2016). Theoretically, all these violations can be associated with pathological synaptic pruning, which emerges as a result of genetic predisposition and environmental influences interaction. All the above studies were mostly conducted after a considerable time after the disease onset, and there is no data on the neurophysiological conditions of occurrence of first psychotic episode in form of hallucinatory-paranoid syndrome.

In our opinion, an important aspect of identifying the pathology of synaptic elimination is the method of investigation of neurophysiological mechanisms and their connection (indirectly through synaptic pruning) with epigenetic functions.

The eliminated synapses are exclusively excitatory contacts (Rakic 1986; Zecevic 1989) and therefore it is considered that synaptic pruning is crucial for establishing the balance of excitation and inhibition processes in the adult cerebral cortex. A well-known fact of schizophrenia disorder is an imbalance of the processes of cortical excitation and inhibition (Foss-Feig 2017), in which the main role belongs to number and quality of synaptic contacts.

The most effective method of analyzing the physiological mechanisms of excitation and inhibition is the method of evoked potentials of the brain, which operates the precise neurophysiological parameters that are closely related to synaptic connectivity: ERP parameters- latency and amplitude.

Assessment of the violation of excitation and inhibition processes interaction in different cortical areas is carried out by ERP method when stimuli of different significance are presented. It is known that in patients with schizophrenia, comparing to the norm, there is a decrease in the amplitude or an increase in the latency of ERP components in frontal areas, which is called hypofrontality (Hill 2004). Most of scientific data witness the predominance of inhibition process in this disease (Lisman 2012). A significant factor is the difference in activation between cortical areas when perceiving stimuli of different significance. In case of excessive rise of stimulus intensity, a state develops that Pavlov called 'overall (protective) inhibition' (1960). However, there are studies that testify an amplitude increase and latency decrease in ERP components, that is the predominance of excitation processes in brain cortex in patients (Overbeek 2019). Such data contradiction may be due to clinical state of patients and neuroleptic therapy. In our studies (Arkhipov 2018) patients were in acute psychotic state were engaged and at the moment of the experiment they haven't receive neuroleptic therapy. Inconsistency can also be explained by limitations of data obtainment in the experiment: studies built separately on the analysis of latency and amplitude may differ in their results.

The ERP method is a non-invasive method evaluating neurophysiological parameters that reflect the state and changes in synaptic connections that determine elimination process.

This article describes a new factor which can counteract to judge the prevalence of one of the main cortical processes (excitation/inhibition) in subjects with paranoid schizophrenia. This is a paradox effect (PE) - simultaneous increase either decrease of both ERP parameters - latency and amplitude. PE 
is an incompatible conjunction/combination of informational processing acceleration with the decrease of neuron amount and, on the contrary, informational processing slow-up with neuron amount decline.

In our work we have studied ERPs to the stimuli of different emotional significance and revealed in patients with paranoid schizophrenia the PE. Emotional significance is caused by the high activation of almost all subcortical structures and reflect the earliest psychic process (Alorda 2007).

Feinberg identified in adolescents EEG changes of sleep, which are characterized by a $75 \%$ decrease in peak amplitude of delta wave (Feinberg and Fein 1983). This can be explained as a sign of a brain metabolic rate decrease, which may be associated with a decrease in neural plasticity. Functional imaging studies using single-photon emission computer tomography and positron emission tomography (Rodgers 2016) revealed additional evidence in favor of this assumption, confirming that blood flow indicators show a pattern of development similar to the ratio between age and synaptic density during synaptic elimination process, which was reported by Huttenlocher (1979). The blood flow intensity increases from birth to its maximum at 5 years (which was $70 \%$ higher than the adult norm) decreased to the level of adults, which was reached at the age of 15-19 years. Since the glucose metabolism measured by PET is substantially determined by synaptic processes activity, it can be considered as a correlate of synaptic density. It is believed that age-related blood flow patterns reflect postnatal development of the brain, accompanied by myelination and dendrite sprouting, followed by synaptic pruning processes. Using the ERP method, Courchesne found (1977) a remarkable decrease of P300 latency from the age of 6 to 23 years(Courchesne). At the same time, it was found that P300 amplitude shows a temporal dynamic similar to synaptic density indices, with maximum values in 4-6 years and a further decrease in later childhood and adolescence (Courchesne 1987). Latency decrease was presumably associated with a myelination increase, while the P300 amplitude was considered to reflect a decrease in the number of synapses.

It is supposed that disturbances seen in hallucinatory-paranoid syndrome are caused by deviations in nervous system ontogenesis at the time of neural connections formation at macro and micro levels as a result of damage of mechanism determining the timing and proper level of compliance of processes necessary for brain development (Lewis 2002). In this regard, it was proposed to study epigenetic disorders that can affect the anlage of neural structures, and further deviations in their physiology and interconnection.

The violation of synaptic elimination at the molecular-genetic level is associated with the pathological expression of synaptic proteins affecting quality and quantity of forming synapses (Wiggin 2005). We studied the methylation of RELN gene encoding the extracellular matrix protein reelin, which plays a trophic role and performs a signaling function, that is, controls the formation of neural systems and regulates synaptogenesis (Bosch 2016). Reelin is produced by neurons and serves as a signaling molecule for the formation of connections between them; its concentration is critical for germination of neural sprouts. When the methylation of RELN gene is impaired, targeting of neural connections (axon/dendrite guidance and target directions) is distorted, leading to indirect connections (Kon 2017). 


\section{Materials and methods}

\section{Subjects}

The study was conducted at psychiatric hospital (Department of acute psychosis). The group of patients included 45 people ( 25 men and 20 women) suffering from first psychotic episode of schizophrenia with a predominance of hallucinatory-paranoid syndrome aged from 18 to 31 years. The diagnosis was made by psychiatrists of the Department of acute psychosis, according to the International Classification of the Disease (ICD) - 10 category F20.0. Total score of severity of psychopathological symptoms determined by the PANSS scale, in patients was $75.2 \pm 6.3$ (Table 1). All patients had their first psychotic attack and did not receive neuroleptic antipsychotic treatment, before and during the experiment. The control group consisted of 43 healthy subjects ( 23 men, 20 women) aged 20 to 32 years. Subjects recruited to both patient and control groups were somatically healthy, right-handed people with $100 \%$ or $100 \%$ corrected vision without substance abuse in medical history. There were no statistically significant differences in gender, age, or educational level between groups $(\mathrm{p}>0.1)$.

Table 1 Clinical Demographics and Characteristics

\begin{tabular}{llll}
\hline Characteristic & Controls $(\mathbf{n}=\mathbf{4 3})$ & Patients $(\mathbf{n = 4 5})$ & $\begin{array}{l}\text { Significance } \\
T \text { Value }\end{array}$ \\
\hline Age (years) & 30.55 & 28.39 & 1.28 \\
\hline Sex: male/female & $23 / 20$ & $25 / 20$ & 1.13 \\
\hline Education: higher/secondary & $34 / 7$ & $37 / 8$ & 1.09 \\
\hline Employment: works/studies & $40 / 3$ & $39 / 6^{*}$ & 1.26 \\
\hline PANSS positive symptoms & - & $30.5 \pm 2.8$ & - \\
\hline PANSS negative symptoms & - & $9.1 \pm 2.3$ & - \\
\hline PANSS general psychopathology & - & $35.9 \pm 4.2$ & - \\
\hline PANSS total psychopathology & - & $75.2 \pm 6.3$ & -
\end{tabular}

Note: PANSS - Positive and Negative Syndrome Scale; Significant $-T$ statistic for the between-group $t$ test; ${ }^{*}$ - at the time of hospitalization.

\section{Electrophysiological methods}

\section{Stimuli and methods of presentation}

Equal number of neutral and threatening images from the International Affective Picture System (IAPS) were selected.

Order of stimuli presentation was random. Time of stimulus presentation was $800 \mathrm{~ms}$, interstimulus interval randomly varied from 1.5 to 3 seconds. 


\section{Procedure}

Subjects of both groups were presented with stimuli on a 14-inch monitor screen at a distance of $0.75 \mathrm{~m}$ from the subject sitting in a dark silent room. Experiment was conducted in implicit conditions.

ERP Recording. EEG was recorded using a 24-channel amplifier MBN (Russia) with 19 leads: Fp1, Fp2, F3, F4, F7, F8, C3, C4, T3, T4, T5, T6, P3, P4, O1, O2, Fz, Cz, Pz (modified 10-20\% electrode placement scheme) with built-in $\mathrm{Ag} / \mathrm{AgCl}$ electrodes. A combined ear electrode was used as a reference lead, with ground electrode located in the center of the forehead. The quantization frequency was $200 \mathrm{~Hz}$, the impedance kept up below $10 \mathrm{kOhm}$.

\section{Data processing and analysis}

After oculographic artifacts remove and excluding epochs with myographic, motor, and other artifacts, filtering was performed. To suppress the electrooculogram, pulse and other artifacts, a method combining EEG filtering and result processing using factor analysis (principal component method) was implemented. High-pass filters were set to $50 \mathrm{~Hz}$, time constant was $0.3 \mathrm{~s}$, quantization frequency was $200 \mathrm{~Hz}$, and impedance was below $10 \mathrm{kOhm}$ (in most recordings $<5 \mathrm{kOhm}$ ). After averaging of evoked potentials across groups and selecting components of interest, the maximum amplitude extremes were found in individual potentials with a step of $5 \mathrm{~ms}$, most close to average ERP in intervals from 170 to 250 ms for the P200 component; from 270 to $340 \mathrm{~ms}$ for the P300 component; and from 370 to $440 \mathrm{~ms}$ for N400. The pre-stimulus interval was $200 \mathrm{~ms}, 300 \mathrm{~ms}$, and $400 \mathrm{~ms}$; after stimulus - $700 \mathrm{~ms}$. For statistical analysis latency and amplitude of P200, P300, and N400 components from all leads were exported.

The results were analyzed using STATISTICA 8.0 software. The Mann-Whitney test was used to verify the experimental hypothesis relating the presence of intergroup differences, and the Wilcoxon test was used to analyze intra-group differences (for two experimental conditions). Exclusively leads with significant $(\mathrm{p}<0.05)$ differences in both amplitude and latency of P200, P300, and N400 were considered. This allowed not to use the correction for multiple comparisons (with 19 leads $0.05 * 0.05 * 19$ $<0.05$, that is, all the considered differences were automatically valid, considering the Bonferroni correction).

\section{Molecular genetic methods}

A panel of DNA extracted from peripheral blood leukocytes was screened for methylation of the RELN gene promoter region. Blood samples were collected in specialized glass tubes with $\mathrm{K}_{3}$ EDTA solution (BD Vacutainer ${ }^{\mathrm{TM}}$ K3EDTA). DNA was purified using hemolysis techniques and magnetic nanoparticles in accordance with the protocols of the companies ('Biret', 'Silex', Russia). Using the latter method, let to acquire RNA together with DNA, suitable for both methylation analysis and analysis of gene expression level by cDNA after reverse transcription and TaqMan RT PCR for RELN gene.

PCR amplification of RELN gene promoter region fragments (about $550 \mathrm{BP}$ ) containing a sequence of $\mathrm{CpG}$ dinucleotides was performed in two stages: first, selected primer pairs were used to amplify a large fragment (450 BP), then a shorter fragment within this sequence (320 BP) was amplified. PCR conditions: $94^{\circ} \mathrm{C} / 4 \mathrm{~min} \times 1$ cycle; $94^{\circ} \mathrm{C} / 1 \mathrm{~min}, 61^{\circ} \mathrm{C} / 2 \mathrm{~min}, 72^{\circ} \mathrm{C} / 2 \mathrm{~min} \times 5$ cycles; $94^{\circ} \mathrm{C} / 1 \mathrm{~min}$, $61^{\circ} \mathrm{C} / 1.5 \mathrm{~min}, 72^{\circ} \mathrm{C} / 1.5-2 \mathrm{~min} \times 25$ cycles; $72^{\circ} \mathrm{C} / 5 \mathrm{~min} \times 1$ cycle . 
Methylation was studied by bisulfate transformation of DNA samples. Bisulfate transformation was performed with 1-2 mcg of DNA treated with bisulfate with minor changes. The DNA was denatured with $0.3 \mathrm{~N} \mathrm{NaOH}$ at $37^{\circ} \mathrm{C}$ for 15 minutes. $15 \mathrm{mcL}$ of freshly prepared $10 \mathrm{mM}$ hydroquinone were added into the test tubes with DNA samples, the solutions were thoroughly mixed using inversion with minimal aeration, $250 \mathrm{mcL}$ of freshly prepared sodium bisulfate $3.6 \mathrm{M}, \mathrm{pH} 5.0$ were added to the test tubes, incubated at $37^{\circ} \mathrm{C}$, and the solutions were thoroughly mixed again using inversion with minimal aeration. The reaction mixtures were coated with mineral oil and incubated at $55^{\circ} \mathrm{C}$ for 16 hours in the dark. The water phases were transferred to new test tubes and desalted on columns with ion exchange resin. DNA was eluted with $120 \mathrm{mcL}$ of distilled water, and traces of alcohol were removed by centrifugation. 10 mcL of $3 \mathrm{~N} \mathrm{NaOH}$ were added to $100 \mu \mathrm{l}$ of desalted DNA and incubated for $15 \mathrm{~min}$ at $37^{\circ} \mathrm{C}$. DNA was precipitated with $33 \mu 110 \mathrm{M}$ of sodium acetate $\mathrm{pH} 7.8$ and $300 \mu \mathrm{l}$ of chilled ethanol using glycogen as a co-precipitator in an ice bath for $10 \mathrm{~min}$, then centrifuged at $13000 \times \mathrm{g}$ for $60 \mathrm{~min}$. The precipitate was resuspended in $100 \mu$ with TE $\mathrm{pH}$ 8.0.

The resulting bisulfite-transformed DNA preparations were subjected to the 'nested' PCR procedure, using specific priming to the above-mentioned regions of the gene promoter region containing GC pairs. The resulting amplifications were cleared of excess primers and non-specific fragments by electrophoresis in 2\% agarose, and then the strips cut from the gel were transferred to sequencing of the corresponding RELN gene promoter regions (Syntol, RF).

\section{Results}

Intra-group comparison of P200, P300, and N400 ERP components to emotionally negative (threatening) stimuli comparing to neutral ones revealed the following statistically significant results:

In healthy subjects, latency of P200 was shorter to threatening stimuli comparing to neutral in right occipital (O2: p < 0.001) (Fig. 1A), right posttemporal (T6: $\mathrm{p}<0.01)$, and right parietal $(\mathrm{P} 4: \mathrm{p}<$ 0.01 ) areas; amplitude of this wave to threatening stimuli was greater than to neutral ones in the same regions. Thus, in healthy people, activation process to emotionally negative stimuli is stronger than to neutral in right occipital, right posttemporal, and right parietal areas.

In patients, latency of P200 component was lower and amplitude higher to threatening stimuli compared to neutral, in right occipital (O2: p < 0.01) (Fig. 1B) and right posttemporal (T6: p < 0.01) regions as well as in the norm. Thus, in patients, as well as in healthy subjects there was a higher activation in the right occipital and right posttemporal regions to emotionally significant stimuli.

In patients in left central area $(\mathrm{C} 3: \mathrm{p}<0.01)$, there was a PE in form of simultaneous decrease of both- latency and amplitude. This PE with a decrease of both P200 parameters does not correspond either to activation or inhibition of the process to stimuli of different significance.

In left lower frontal area (F7: $\mathrm{p}<0.01)$, a PE was found in form of simultaneous increase of P200 latency and amplitude, which is also not corresponding to the dynamics of activation to stimuli of higher significance.

In healthy subjects, latency of P300 component was lower to threatening stimuli comparing to neutral in left occipital (O1: p < 0.01), left posttemporal (T5: p $<0.001)$, left central (C3 p $<0.01)$, right central $(\mathrm{C} 4: \mathrm{p}<0.05)$, and right lower frontal $(\mathrm{F} 8: \mathrm{p}<0.01)$ areas; amplitude of this component in the 
same regions to threatening stimuli was greater than to neutral ones. Thus, in healthy individuals, activation to emotionally negative stimuli were greater than to neutral in left occipital, left posttemporal, left central, right central, and right lower frontal regions.

In patients latency of P300 component was lower, and amplitude greater to threatening stimuli comparing with neutral, as well as in healthy controls in left occipital (O1: $p<0.01)$ and left lower temporal $(\mathrm{T} 5: \mathrm{p}<0.01)$ areas. Thus, in patients with emotionally significant stimuli, higher activation to threatening stimuli is observed, as well as in healthy people in the left occipital and left lower temporal regions.

In patients in P300 component there was found PE same as in P200 component, in form of simultaneous decrease of both amplitude and latency in midcentral $(\mathrm{Cz}: \mathrm{p}<0.01)$ (Fig. 2A) and right central $(\mathrm{C} 4: \mathrm{p}<0.01)$ areas; this PE in P300 component also does not correspond to activation dynamics to stimuli of different significance.

In left prefrontal (Fp1: p < 0.01) (Fig. 2B), right prefrontal (Fp2: p < 0.01), left lower frontal (F7: p < $0.01)$ and right lower frontal (F8: p < 0.01) areas, patients showed a PE, as well as in P200 component, in form of simultaneous increase in both ERP parameters. This PE in P300 component also does not correspond to activation dynamics to stimuli of different significance.

In healthy subjects, latency of N400 component was shortened to threatening stimuli compared to neutral in right posttemporal (T6: $\mathrm{p}<0.01)$, right prefrontal (Fp2: $\mathrm{p}<0.01)$, and midcentral $(\mathrm{Cz}$ : $\mathrm{p}<$ 0.01) regions; amplitude of this component in the same regions to threatening stimuli was greater than to neutral ones. Thus, in healthy individuals, higher activation to emotionally significant stimuli is noted in right posttemporal, left prefrontal, and midcentral areas.

In patients latency of N400 component was lower, and amplitude greater to threatening stimuli comparing to neutral, same as in healthy subjects in right posttemporal (T6: $p<0.01)$ area. Thus, in patients as well as in norm activation to emotionally significant stimuli were found in right posttemporal area.

In midcentral $(\mathrm{Cz}: \mathrm{p}<0.01)$ and right central $(\mathrm{C} 4: \mathrm{p}<0.01)$ regions, patients had a PE, as well as in the P200 and P300 components, in form of simultaneous decrease of both ERP parameters. This PE in N400 wave does not correspond to activation dynamics to stimuli of different significance.

In patients in N400 as well as in P200 and P300 components, PE was observed, in form of simultaneous both ERP parameters increase in right prefrontal $(\mathrm{Fp} 2: \mathrm{p}<0.01)$ and right lower frontal (F8: $\mathrm{p}<0.01)$ areas. This PE in N400 component also does not correspond to activation dynamics to stimuli of different significance.

\section{Results of RELN methylation}

Analysis of methylation of RELN gene obtained from peripheral blood (promoter region -600 to 900), site -442 , revealed $100 \%$ absence of methylation in patients with schizophrenia (Fig. 3). In healthy individuals, $99 \%$ of RELN methylation in this promoter region is present in all CG pairs. A consequence of RELN gene demethylation in patients is an increase of reelin protein expression. 


\section{Discussion}

The aim of the study was to identify the neurophysiological mechanisms of psychosis in schizophrenia and to consider the relationship between two levels - physiological (based on the analysis of ERP to highly significant and neutral stimuli) and molecular genetic (analysis of methylation of RELN gene sites), to examine the relationship of these levels to define the etiopathogenetic factor of hallucinatoryparanoid syndrome.

In our study, the highest ERP brain activation response to significant stimuli was found in P200, P300, and N400 components. The fact that these components respond to negative stimuli with increased activation (latency shortening and amplitude increase) in both groups of subjects and in the same areas (occipital and posttemporal) (Fig. 1) indicates that the processing of primary visual information in sensory areas in schizophrenia is preserved.

Differences appear $200 \mathrm{~ms}$ after stimulus presentation. It is a pathology found in patients' neural networks, which manifests in a simultaneous increase of both ERP parameters - latency and amplitude in frontal and anterior temporal areas and their simultaneous decrease in central areas. Both simultaneous increase and decrease of amplitude and latency form /conceive/ constitute PE from physiological point of view which can be seen in patients with schizophrenia. The detection of PE is the most important results of our study, as it shows for the first time the incongruence of ERP parameters - a simultaneous increase in both amplitude and latency, or their simultaneous decrease in response to significant stimuli in certain areas in schizophrenia. Simultaneous unidirectional shift in both amplitude and latency in response to significant stimuli in certain areas in schizophrenia.

Appearance of PE in the P200 component, which is called the recognition potential, is associated with orientation response (Rozynski 2015), information synthesis (Ivanitsky 1995), and emotional evaluation of stimuli ( $\operatorname{Raz} 2013$ ) indicates a violation of these functions in patients with schizophrenia, presenting mainly in frontal and central regions. PE in P300 component, registered in the same areas as P200, indicates a violation of emotional assessment of significance and the decision-making process based on 'information synthesis' in schizophrenia (Polich 2007). PE in N400 component in prefrontal regions in patients with schizophrenia can be explained by a violation of matching information with past experience and correcting of stimulus perception (Curran 1993).

The PE in ERP in patients cannot be defined neither as the process of excitation nor inhibition, it must be probably a conflict of these mechanisms. This abnormal state is caused by synaptic pruning violation, which manifests in ERP in two ways. First - in a simultaneous decrease in latency and amplitude, and second - in an increase of both these parameters. PE with latency and amplitude reduction (Fig. 2A) is probably a consequence of smaller number of neurons activated by the stimulus (decrease in amplitude), and smaller number of synapses (decrease in latency) due to excessive pruning. PE with latency and amplitude increase (Fig. 2B) can be seen because of increase of responding neurons number (increase of amplitude), but time (peak latency) also increases due to a larger number of synapses activating neurons. However, these synapses, due to pathological pruning, remain functionally defective, aberrant, that is, both number and quality of synapses alteration takes place. 
It is important to note that the process of synaptic pruning extends from occipital areas, through central ones to frontal regions, which may be explained by the evolutionary and ontogenetic dynamics of nervous system development (Huttenlocher 1997).

Apparently, in patients before the psychosis onset, there is a bigger number of synapses in central cortical areas, and by the time of pruning process beginning in frontal areas, number and functional state of synapses in central-parietal areas is already disrupted. At the same time, the process of synapse elimination taking place in frontal regions is insufficient, which finally leads to heterogeneity of elimination and synaptic inhomogeneity in frontal and central regions. On this basis, we assume that in schizophrenia a decline of synapse number takes place, but for the conditions of the first psychotic episode occurrence and for predominance of productive symptoms in clinical picture, it is necessary not only an inhomogeneous ratio of number of synapses in frontal and central cortex, but also a greater number of aberrant synapses in frontal regions, which in turn, during the course of the disease, become inactivated with low elimination, which eventually entails to a gray matter density decrease in schizophrenia with predominance of negative symptoms in the clinical picture.

Since patients in psychosis, in contrast to norm, demonstrated the presence of PEs, it can be asserted that elimination heterogeneity forming synaptic inhomogeneity is a prerequisite for the psychosis occurrence, and the distribution of heterogeneity of elimination is such that the number of synapses in central cortical regions declines, and in the frontal regions - increases due to aberrant synapses.

The process of elimination of excessive synapses - pruning takes place mainly in puberty and normally engages about $40 \%$ of total synapses number. In ontogenesis, only those links that are involved in functional units or circuits become permanent, while those that are not involved become inactive and eventually resorbed (Huttenlocher 1994). The most liable basic physiological mechanism of neural connections formation is competition for a signal protein that acts as a target molecule for synapses and dendrites (Faigle 2013).

Process of pruning of excessive synaptic connections is determined by work of extracellular matrix regulatory protein - reelin, whose expression depends on the level of methylation of gene encoding this protein.

The fact that in our studies all patients were found to have demethylation of RELN gene in peripheral blood (promoter and site) corresponds to an increase of reelin expression in blood in patients comparing to norm. It is known that in schizophrenia there is a reciprocal ratio of methylation level in peripheral blood and brain tissue (Walton 2016). Thus, we can talk about hypermethylation of RELN gene in the brain and, accordingly, a decrease of reelin expression.

It is known that promoter region includes binding sites of such factors as CREB (cyclic AMP response element-binding protein) - one of the factors that determine transcription and expression of genes that play a crucial role in nervous system development and functioning (Steven 2020). This fact confirms the functional importance of detected methylation disturbance in $\mathrm{CpG}$ dinucleotide of -422 site adjacent to CREB binding area (Fig. 3).

Reelin is a target molecule for guidance of synaptic connections during their formation (Niu 2008). Due to the lack of reelin, the competition of synapses for the target molecule (which in our opinion is a key mechanism) leads to aberrant anlage of these connections and insufficient resorption of synapses. 
Result of the latter is an emergence of excessive number of synapses with the violated functions of information transmitting and processing. This number gradually increases which is reflected in the conflict of excitation and inhibition processes in form of PE in ERP. Our conclusion doesn't contradict the data of MRI studies that describe a decrease of gray matter density (Venkatasubramanian 2008; Mørch-Johnsen 2017), since we assume that the decrease in density can emerge because of insufficiently resorbed synaptic spines, not with a decrease in their number.

It is worth to note the inequality of the pruning process, which first takes place in central and parietal areas and gradually passes to frontal ones. While synaptic deficit increases in central regions, a number of ill-functioned synapses emerge in frontal areas. Insufficiently resorbed synapses stop functioning, which can be detected ad simultaneous shortening of latency and an amplitude decrease in ERP components forming PE. At the same time, an excessive number of insufficiently resorbed synapses with aberrant functioning takes place in frontal regions, with PE manifesting in simultaneous increase of both ERP latency and amplitude. Heterogeneity of elimination leads to inhomogeneity of synapses in different cortical areas, which we consider as a crucial condition for the first psychotic episode onset in schizophrenia.

\section{Acknowledgements}

The authors would like to thank Rodionov Georgiy Iliych for his help with translation the manuscript and logistics of materials for the experiment.

\section{Funding}

No funding was received to assist with the preparation of this manuscript.

\section{Competing interests}

The authors have no conflicts of interest to declare that are relevant to the content of this article.

\section{Ethics approval}

All procedures performed in studies involving human participants were in accordance with the ethical standards of the institutional and/or national research committee and with the 1964 Helsinki Declaration and its later amendments or comparable ethical standards.

\section{Consent to participate}

Informed consent was obtained from all individual participants included in the study.

\section{Data availability}

The data that support the findings of this study are available from the corresponding author, upon reasonable request. 


\section{References}

Alorda C, Serrano-Pedraza I, Campos-Bueno JJ, Sierra-Vazquez V, Montoya P (2007) Low spatial frequency filtering modulates early brain processing of affective complex pictures. Neuropsychologia. 45(14):3223-3233.

Arkhipov AY, Novototsky-Vlasov VYu, Nurbekov MK, Strelets VB (2018) [The influence of negative emotional stimuli on the late perception stages (P300 \& N400 components) in patients with paranoid schizophrenia in implicite situation]. Zhurnal Vysshei Nervnoi Deiatelnosti Imeni I.P. Pavlova. 68(1):28-40.

Borgwardt SJ, McGuire PK, Aston J, Gschwandtner U, Pfluger M, Stieglitz R, Radue E, Riecherrossler A (2008) Reductions in frontal, temporal and parietal volume associated with the onset of psychosis. Schizophrenia Research. 106(2-3):108-114.

Bosch C, Masachs N, Exposito-Alonso D, Martínez A, Teixeira CM, Fernaud I, Pujadas L, Ulloa F, Comella JX, DeFelipe J, Merchán-Pérez A, Soriano E (2016) Reelin regulates the maturation of dendritic spines, synaptogenesis and glial ensheathment of newborn granule cells. Cerebral Cortex. 26(11):4282-4298.

Courchesne E, Elmasian R, Yeung-Courchesne R. (1987) Electrophysiological correlates of cognitive processing: P3b and Nc, basic, clinical, and developmental research. In: Halliday AM, Butler SR, Paul R, eds. A textbook of clinical neurophysiology. New York: Wiley. 645-676.

Courchesne E (1977) Event-related brain potentials: comparison between children and adults. Science. 197(4303):589-592.

Curran T, Tucker DM, Kutas M, Posner MI (1993) Topography of the N400: brain electrical activity reflecting semantic expectancy. Electroencephalography and Clinical Neurophysiology. 88(3):188-209.

Faigle R, Song H (2013) Signaling mechanisms regulating adult neural stem cells and neurogenesis. Biochim Biophys Acta. 1830(2):2435-2448.

Feinberg I, Fein G, Floyd TC, Aminoff MJ (1983) Delta (0.5-3 Hz) EEG waveforms during sleep in young and elderly normal subjects. In: Chase MH and Weitzman ED eds. Sleep Disorders: Basic and Clinical Research. Spectrum, New York. 449-462.

Feinberg I (1983) Schizophrenia: caused by a fault in programmed synaptic elimination during adolescence? J Psychiatr Res. 17(4):319-334.

Feinberg I (1990) Cortical pruning and the development of schizophrenia. Schizophrenia Bulletin. 16(4):567-570.

Foss-Feig JH, Adkinson BD, Ji JL, Yang G, Srihari VH, McPartland JC, Krystal JH, Murray JD, Anticevic A (2017) Searching for cross-diagnostic convergence: neural mechanisms governing excitation and inhibition balance in schizophrenia and autism spectrum disorders. Biological psychiatry. 81(10):848-861.

Fromer M, Roussos P, Sieberts S, Johnson JS, Kavanagh DH, Perumal TM, Ruderfer DM, Oh EC, Topol A, Shah HR, Klei LL, Kramer R, Pinto D, Gümüş ZH, Cicek AE, Dang KK, Browne A, Lu C, Xie L, Readhead B, Stahl EA, Xiao J, Parvizi M, Hamamsy T, et al. (2016) Gene expression 
elucidates functional impact of polygenic risk for schizophrenia. Nat Neurosci. 19(11):14421453.

Glausier JR, Lewis DA (2013) Dendritic spine pathology in schizophrenia. Neuroscience. 251:90-107.

Higley JR, Walker MA, McDonald B, Crow TJ, Esiri MM (2003) Size of hippocampal pyramidal neurons in schizophrenia. British journal of psychiatry. 183(4):414-417.

Hill K, Mann L, Laws KR, Stephenson CME, Nimmo-Smith I, McKenna PJ (2004) Hypofrontality in schizophrenia: a meta-analysis of functional imaging studies. Acta Psychiatr Scand. 110(4):243256.

Hoffman RE, Dobscha SK (1989) Cortical pruning and the development of schizophrenia: a computer model. Schizophr Bull. 15(3):477-490.

Huttenlocher PR, Dabholkar AS (1997) Regional differences in synaptogenesis in human cerebral cortex. Journal of comparative Neurology. 387(2):167-178.

Huttenlocher PR (1979) Synaptic density in human frontal cortex - developmental changes and effects of aging. Brain Res. 163(2):195-205.

Huttenlocher PR (1994) Synaptogenesis, synapse elimination, and neural plasticity in human cerebral cortex. In Threats to Optimal Development: Integrating Biological, Psychological, and Social Risk Factors. In: Nelson CA eds. New Jersey, Hillsdale Lawrence Erlbaum associates publishers. 27:35-54.

Ivanitsky AM (1995) Information synthesis in cortical areas as an important link in brain mechanisms of mind. Behavioral and Brain Sciences. 18(4):686-687.

Jernigan TL, Zisook S, Heaton RK, Moranville JT, Hesselink JR, Braff DL (1991) Magnetic resonance imaging abnormalities in lenticular nuclei and cerebral cortex in schizophrenia. Arch Gen Psychiatry. 48(10):881-890.

Keshavan MS, Anderson S, Pettegrew JW (1994) Is schizophrenia due to excessive synaptic pruning in the prefrontal cortex? The Feinberg hypothesis revisited. J Psychiatr Res. 28:239-265.

Kon E, Cossard A, Jossin Y (2017) Neuronal polarity in the embryonic mammalian cerebral cortex. Frontiers in Cellular Neuroscience. 11.

Lewis DA, Levitt P (2002) Schizophrenia as a disorder of neurodevelopment. Annu Rev Neurosci. 25:409-432.

Lisman J (2012) Excitation, inhibition, local oscillations, or large-scale loops: what causes the symptoms of schizophrenia? Current opinion in neurobiology. 22(3):537-544.

Mauney SA, Pietersen CY, Sonntag K-C, Woo T-UW (2015) Differentiation of oligodendrocyte precursors is impaired in the prefrontal cortex in schizophrenia. Schizophrenia Research. 169(13):374-380.

Mørch-Johnsen L, Nesvaag R, Jørgensen KN, Lange EH, Hartberg CB, Haukvik UK, Kompus K, Westerhausen R, Osnes K, Andreassen OA, Melle I, Hugdahl K, Agartz I (2017) Auditory cortex characteristics in schizophrenia: associations with auditory hallucinations. Schizophrenia Bulletin. 43(1):75-83.

Niu S, Yabut O, D'Arcangelo G (2008) The reelin signaling pathway promotes dendritic spine development in hippocampal neurons. Journal of Neuroscience. 28(41):10339-10348. 
Overbeek G, Gawne TJ, Reid MA, Salibi N, Kraguljac NV, White DM, Lahti AC (2019) Relationship between cortical excitation and inhibition and task-induced activation and deactivation: a combined magnetic resonance spectroscopy and functional magnetic resonance imaging study at $7 \mathrm{~T}$ in first-episode psychosis. Biological psychiatry. Cognitive neuroscience and neuroimaging. 4(2):121-130.

Pavlov IP (1960) Conditioned reflex: An investigation of the physiological activity of the cerebral cortex. New York, NY: Dover Publications Inc.

Polich J (2007) Updating P300: An integrative theory of P3a and P3b. Clinical Neurophysiology. 118(10):2128-2148.

Rakic P, Bourgeois J-P, Eckenhoff MR, Zecevic N, Goldman-Rakic PS (1986) Concurrent overproduction of synapses in diverse regions of the primate cerebral cortex. Science. 232(4747):232-235.

Raz S, Dan O, Arad H, Zysberg L (2013) Behavioral and neural correlates of emotional intelligence: an event-related potentials (ERP) study. Brain Res. 1526:44-53.

Rodgers ZB, Detre JA, Wehrli FW (2016) MRI-based methods for quantification of the cerebral metabolic rate of oxygen. Journal of Cerebral Blood Flow and Metabolism. 36(7):1165-1185.

Rozynski MM, Chen C (2015) Electrophysiological changes in P200 latency and amplitude of jittered orientation visual integration task in healthy participants: a multi-block design EEG study. Honors Scholar theses, University of Connecticut.

Steven A, Friedrich M, Jank P, Heimer N, Budczies J, Denkert C, Seliger B (2020) What turns CREB on? And off? And why does it matter? Cell Mol Life Sci. 77(20):4049-4067.

Venkatasubramanian G, Jayakumar PN, Gangadhar BN, Keshavan MS (2008) Automated MRI parcellation study of regional volume and thickness of prefrontal cortex (PFC) in antipsychoticnaive schizophrenia. Acta Psychiatr Scand. 117(6):420-431.

Walton E, Hass J, Liu J, Roffman JL, Bernardoni F, Roessner V, Kirsch M, Schackert G, Calhoun V, Ehrlich S (2016) Correspondence of DNA methylation between blood and brain tissue and its application to schizophrenia research. Schizophrenia Bulletin. 42(2):406-414.

Wannan C, Cropley VL, Chakravarty MM, Bousman C, Ganella RP, Bruggemann JM, Weickert TW, Weickert CS, Everall I, McGorry P, Velakoulis D, Wood SJ, Bartholomeusz CF, Pantelis C, Zalesky A (2019) Evidence for network-based cortical thickness reductions in schizophrenia. Am J Psychiatry. 176(7):1-12.

Wiggin GR, Fawcett JP, Pawson T (2005) Polarity proteins in axon specification and synaptogenesis. Developmental Cell. 8(6):803-816.

Zecevic N, Bourgeois J-P, Rakic P (1989) Changes in synaptic density in motor cortex of rhesus monkey during fetal and postnatal life. Dev Brain Res. 50(1):11-32. 
Fig. 1 Activation in the occipital areas in schizophrenia patients and healthy control

Notes: ERP results for healthy control (Nor) (A) and patients (SCH) (B) in right occipital area (O2). These include grand-averaged P200 ERP waveforms in response to significant (dark line) and neutral (light line) stimuli over occipital sites.

Fig. 2 Paradox effects of parameters of evoked potentials in schizophrenia patients

Notes: ERP results for schizophrenia patients (SCH): A) PE with decrease of both P300 parameters in midcentral area $(\mathrm{Cz})$; B) PE with increase of both P300 parameters in left prefrontal area (Fp1). A) Grand-average P300 waveforms in response to significant (dark line) and neutral (light line) stimuli over midcentral area; B) the same over left prefrontal area.

Fig. 3 RELN methylation in schizophrenia

Notes: Numbers indicate the positions of termini from 5' to 3' - respectively from the transcription start site (TSS).

TGACGTCC - (highlighted in red) - CREB factor binding site.

is the transcription start site, in the following the region of the first exon is highlighted (Ensemble database).

CG - significant methylation site -442 .

GGCTCAGCGGTCCTCGACAGCGTCCCCGT - rcr4 - sequencing primer.

$(\square)$ - cN-myc transcription factor binding site.

(1) - Tbr1 transcription factor binding site. 


\section{Figures}

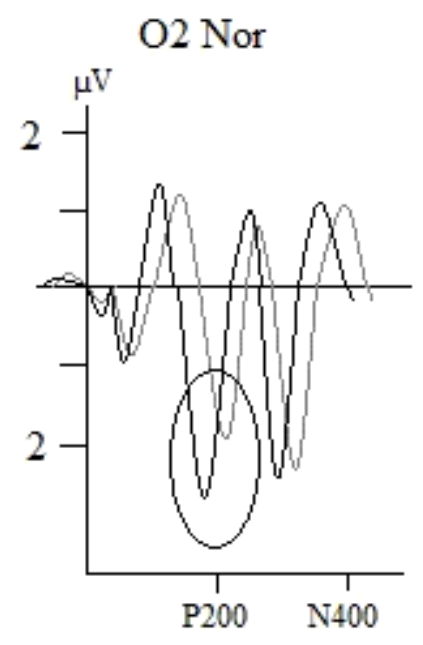

A

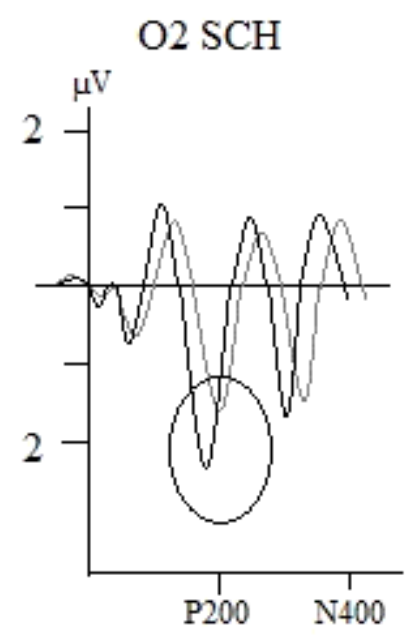

B

Figure 1

Activation in the occipital areas in schizophrenia patients and healthy control. Notes: ERP results for healthy control (Nor) (A) and patients ( $\mathrm{SCH}$ ) (B) in right occipital area (O2). These include grand-averaged P200 ERP waveforms in response to significant (dark line) and neutral (light line) stimuli over occipital sites.

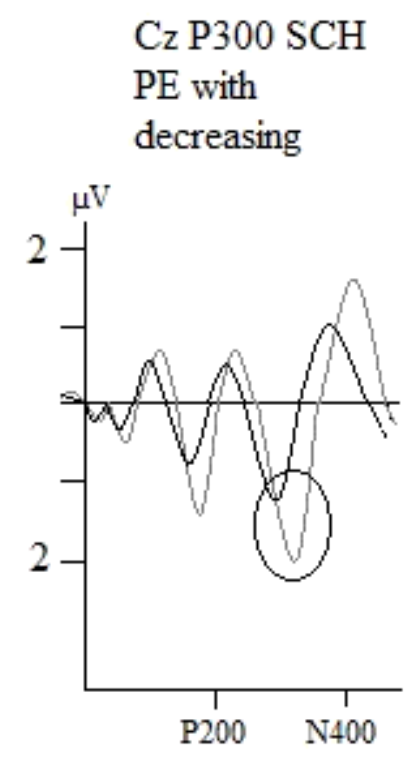

A

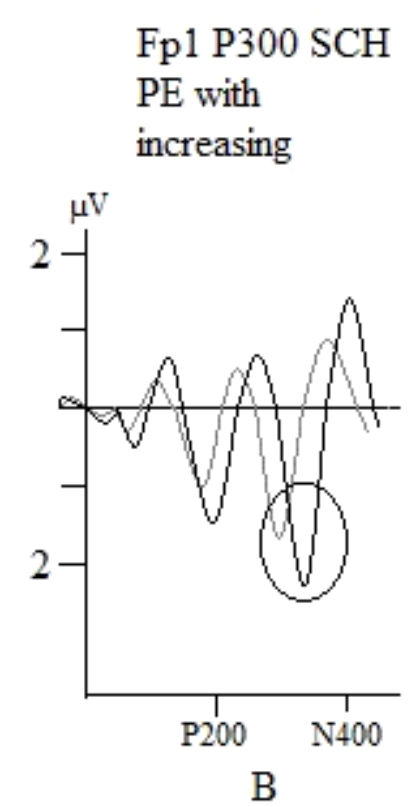

B

\section{Figure 2}

Paradox effects of parameters of evoked potentials in schizophrenia patients. Notes: ERP results for schizophrenia patients (SCH): A) PE with decrease of both P300 parameters in midcentral area (Cz); B) PE with increase of both P300 parameters in left prefrontal area (Fp1). A) Grand-average P300 
waveforms in response to significant (dark line) and neutral (light line) stimuli over midcentral area; B) the same over left prefrontal area.

\begin{abstract}
$(-600)$
5' - CGCGGGACGCAGCCGGCT CAGACAAAGAAACCGGCGCCAGCGGCCGICCCTGCCGCC CCT CTCCTTCCCTCACGCATCCTCCCAGGA.AAAA CAGGGCACACTGACGGCCAAAGGGGC TGGCCTI CCCCT TCAGACAGCTGGA CGGGAGGTGCCCTCTGCGGGGCT TTGACGICCCTC GCAGAAGAGTCGCGGGCTCAGCGGI CCTCGACAGCGICCCCGICCCGCICCCCGGCGGGC GCCCCTCCCTGI CCTCCCGGGTGCGAACCGGGCGCTGGCCGGGGACTCCGGGGACGCGTG CGCCCCT CGCCGCGCGAGGTGCCGCCGAGCCAGCCCGAGAGGGCGGGGGGCGGGCGGGGC GGCGCGCGGGGGCGGGGGAGCGGCCGGGACACGI GIGGCGGCGGCGGGGGGGACGCGGCG CCCGGGGCTTTAAGAA GGTGI GGAGCGGGGCGGGCGCTI TCCCAGGCCIGGCCGAGGGGC GTCGCGCAGAGGCGGCGGCGGCGCACGGAGGCGGCAGACGACGCGCTCTCGGCGCCCGCA GCCCCGGICCCGCGCT CCCGCGGCCCAAAGTAACIIIGGGAGCCGCCGICTCCCGCGGAA CTI TCGGCGGGGGCCCGCTCCCAGGCCCGCTCCOGAGCCCGTTCCGCTCCCGTCCGCCT TCTTCTCGCCTTCTCTCCGCGTGGCTCCTCCGTC AGCGGCGCGTAGGGCGCGCGGCGGCGGCGGCGGCGGCGGCGGCATGGAGCGCAGTGGCTG GGCCCGGCAGACTTTCCTCCTAGCGCTGTTGCTGGGGGCGACGCTGAGGGCGCGCGCGGC GGCTGGCTATTA CCCCCGCTTTTCGCCCTTCTTTTTCCT GTGCACCCA CCACGGGGAGCT GGAAGGGGATGGGGAGCAGGGCGAGGTGCTCATTTCCCTGCATATTGCGGGCAACCCCAC CTACTACGTTCCGGGACAAGAATACCATGGTAGGIACCGCAGCACCICCGCGCICGCCGC CGCCGGGIGCGCCCGC CAGCGCACCIIICI CATCCCIII CCII CTICCAAGGGGCCI IGG AAA CAAGAACCA TGACAACCCTGGCCCCAG CGAT GCGCT TGGI CCAGG GAGCGAGAATAA AGI TAGI GGAGAATGGAGTCA CAAGIIGGGAITCAITCI CCAI GICCCCACI CCCCGAAC GCATCTCCTGGI AATGAGCTGGTGCITGIGCIAAACGCCIGAGAATTCIIGGAGGCCGGI GICITTCCCCCI CACT CCCCGIACT IACCGCAGGCACTI TAAAIIGAACCTGCGCAI III GTI ICTT TACTCCTAGGACTI GGAI IICAGACCI IIGICCIGCICCCGGICCCCCCAGAI GGGTCCCCGGGGGGGC GITGGGGGCTGICGCIGGGCIII GCGGGCAGGCIGGIGACA GAI IGAAGCGCCCAGGICT GTGGCIGCCCCGCGCCGCTCGACCGCAAAGII CCCGCIGCI CAC CAG $-3^{\prime}$
\end{abstract}

$(+900)$

\title{
Figure 3
}

RELN methylation in schizophrenia. Notes: Numbers indicate the positions of termini from $5^{\prime}$ to $3^{\prime}-$ respectively from the transcription start site (TSS). TGACGTCC - (highlighted in red) - CREB factor binding site. $\mathrm{C}$ is the transcription start site, in the following the region of the first exon is highlighted (Ensemble database). CG - significant methylation site -442. GGCTCAGCGGTCCTCGACAGCGTCCCCGT - rcr4 - sequencing primer. (_) - cN-myc transcription factor binding site. (_) - Tbr1 transcription factor binding site. 